American Journal of Pharmaceutical Education 2019; 83 (8) Article 7244.

\title{
RESEARCH
}

\section{Student Pharmacists' Use of Patient-Centered Communication Skills During an Introductory Pharmacy Practice Experience}

\author{
Benjamin Teeter, PhD, Rachel Stafford, PharmD, Nalin Payakachat, PhD, Jessica Reid, PharmD, \\ Kaci Thiessen, PharmD, Amy Franks, PharmD, Catherine O’Brien, PharmD \\ University of Arkansas for Medical Sciences College of Pharmacy, Little Rock, Arkansas \\ Submitted June 28, 2018; accepted October 23, 2018; published October 2019.
}

\begin{abstract}
Objective. To assess how Doctor of Pharmacy (PharmD) students use patient-centered communication (PCC) during a community pharmacy introductory pharmacy practice experience (IPPE).

Methods. All first-year student pharmacists at the University of Arkansas for Medical Sciences completed a required course in patient-centered communication that included training in motivational interviewing. After subsequently completing their first IPPE, the students submitted written reflections on their use of PCC during the experience. The reflections were de-identified and template analysis was conducted. With this method, predetermined codes are established, and a constant comparison method is used to finalize overarching themes. The students' overall level of reflection was assessed and each reflection was coded. Discrepancies were resolved through in-depth discussion and negotiated consensus.

Results. Of the 116 student reflections submitted, 951 codes were applied. Six overarching themes were identified: acknowledgement of motivational interviewing skills/components; philosophy of patient-centeredness; barriers to using PCC or motivational interviewing; benefits from use of PCC; demonstration of good general communication skills; and perceptions of patient encounters. Approximately $46 \%$ of students reflected at a level three or higher (ie, personal insight or intended behavior change).

Conclusion. First-year student pharmacists successfully used PCC skills during their IPPE. These findings demonstrate the impact that a PCC course can have on students' overall patient-centeredness and highlights barriers that students struggle to overcome. Other pharmacy schools may benefit from implementing a similar PCC course. Activities that reiterate these skills and provide additional opportunities to practice PCC are necessary throughout the curriculum.
\end{abstract}

Keywords: patient-centered, communication, reflection, motivational interviewing

\section{INTRODUCTION}

Patient-centered care is defined by the Institute of Medicine as the provision of care that is "respectful of and responsive to individual patient preferences, needs, and values, and ensures that patient values guide all clinical decisions." Improvements in patient outcomes, such as self-management, satisfaction, and adherence, have been documented with patient-centered care. ${ }^{2,3}$ Standards two and three of the Accreditation Council for Pharmacy Education's 2016 Standards $^{4}$ and the American Association of Colleges of Pharmacy's Center for the Advancement of Pharmacy Education (CAPE) Educational

Corresponding Author: Benjamin Teeter, College of Pharmacy, University of Arkansas for Medical Sciences, 4301 W. Markham St., Little Rock, AR 72205. Tel: 501-526-5743. E-mail: bsteeter@uams.edu
Outcomes from $2013^{5}$ both emphasize the importance of educating future pharmacists to provide patient-centered care and communicate with patients in a patient-centered way. As the pharmacy profession continues to move toward and embrace the idea of patient-centered care, colleges and schools of pharmacy are increasingly exploring methods to effectively teach their students and ensure they are prepared and capable to communicate with their patients. However, evaluation of teaching strategies to improve students' patient-centered communication (PCC) is an area where research is sparse.

Future pharmacists need a systematic, effective, patient-centered approach to communicating with patients in order to encourage behavior change. Motivational interviewing is one such approach. Motivational interviewing is an evidence-based, patient-centered way of communicating with patients to elicit their own 


\section{American Journal of Pharmaceutical Education 2019; 83 (8) Article 7244.}

motivations for behavior change. ${ }^{6}$ An integral part of the Patient Care Process developed by the Joint Commission of Pharmacy Practitioners (JCPP) is patient communication and empowering patients. Colleges and schools of pharmacy need effective curricular strategies to teach patient-centered communication, such as motivational interviewing, to students. Ensuring that attention is paid to both skill introduction and development, with appropriate practicing of skills in simulated and actual patient care environments, can be challenging during the professional curriculum. Little is known regarding student pharmacists' utilization of communication techniques taught during the didactic curriculum in real-world patient care scenarios. Additionally, few examples from previously published literature describe students' self-reported attempts to use motivational interviewing.

In order to assist future pharmacists in engaging their patients in positive behavior change, Introduction to Patient Centered Communication, a required course for first-professional year (P1) Doctor of Pharmacy (PharmD) students, was developed and implemented. ${ }^{7}$ The course provides training in motivational interviewing and provides the opportunity for students to practice their communication in faculty-facilitated role-play activities. While these role-play experiences provide students with the opportunity to use their newly learned communication techniques, and the skills are assessed and reinforced later in the curriculum, little is known about the use of these skills in real-world situations after the conclusion of the first-year course. Therefore, the objective of this study was to better understand how students use motivational interviewing during their introductory pharmacy practice experience (IPPE) in the community pharmacy setting immediately following the completion of their first didactic year and the Introduction to Patient Centered Communication course. This study analyzed student-written reflections to identify themes related to the uptake of motivational interviewing and explore relationships between discovered themes. Useful information gained from this qualitative evaluation can be used to determine student successes with and barriers to using motivational interviewing skills in their community IPPE, and to direct future curricular refinements in order to enhance PCC in student pharmacists.

\section{METHODS}

This study used a qualitative design to retrospectively explore written reflections of student pharmacists at a public university on the use of motivational interviewing during their community pharmacy-based IPPE in the summer of 2015. The three-week long IPPE exposes students to the community pharmacy setting after the first year of didactic curriculum. After finishing the IPPE, students were required to write and submit a reflection on PCC with specific instructions to describe an example of how they practiced reflecting a patient's core concern (or used other motivational interviewing skills) during the community IPPE. Specifically, they were asked what happened, what did or did not go well, and what they learned. Students submitted their written responses to these questions through Blackboard (Washington, DC) course management software. They were given clear, minimum expectations to describe their attempt to use motivational interviewing and respond to three questions; however, there was no length requirement or limit set. This study was granted exempt status by the institutional review board of the University of Arkansas for Medical Sciences.

All student-written reflections were de-identified and uploaded to MAXQDA Plus 12 (Berlin, Germany) qualitative data analysis software. ${ }^{8}$ Directed content analysis was used to explore barriers and facilitators to using communication skills and motivational interviewing during the IPPE, as well as students' overall feelings towards their experiences. This approach is used to systematically and objectively describe and quantify phenomena extracted from written, verbal, or visual communication messages. ${ }^{9}$ A deductive approach using a mixture of the instructed prompts and other key concepts from motivational interviewing, such as "developing discrepancy" and "rolling with resistance," was used to guide the creation of the initial codebook. Thus, the initial codebook consisted of general codes that could reasonably be expected to appear in the written reflections and would serve as a strong foundation for analysis.

To further develop and finalize the codebook, 20 reflections were analyzed in a three-week run-in period using an inductive approach. The run-in period was also used to train all coders to apply the initial codes consistently and to finalize these code definitions. Each reflection completed during the run-in period was coded by two researchers to clarify existing code definitions and add new codes for topics of interest not covered in the initial codebook. The research team met weekly and used a constant comparison approach to discuss new codes and agree on definitions. All codes were then sorted into themes based on their relationships to one another, and themes were then grouped into meaningful clusters to finalize the codebook. Saturation of themes and subthemes occurred after the three-week run-in period as no new codes or themes emerged.

The final codebook had six overarching themes. Each overarching theme was made up of multiple codes and subcodes. In addition to analyzing themes, overall 


\section{American Journal of Pharmaceutical Education 2019; 83 (8) Article 7244.}

level of reflection was assessed for each reflection using a five-level scheme adapted from the literature. ${ }^{10,11}$ Levels of reflection ranged from 0 (absent, no mention of patientcentered communication in the reflection) to 4 (critical reflection, behavior change/intention to change behavior stated in reflection) to provide an overall impression of students' ability to write a quality reflection. Definitions of each of the five levels can be found in Table 1.

Once the codebook was finalized, each reflection was randomly assigned to two coders. Each coder analyzed reflections independently using the final codebook, with the possibility that each sentence in a reflection could be categorized into multiple codes or subcodes. To ensure reliability and validity of the qualitative content analysis, investigators met weekly to resolve discrepancies through in-depth discussion between the two coders. If discrepancies could not be resolved between the two coders, other research team members offered their opinions until agreement was achieved. Descriptive statistics and a detailed explanation of the utterances that made up each theme are presented to provide indepth understanding of the phenomena.

\section{RESULTS}

All of the student pharmacists $(\mathrm{N}=116)$ completed a reflection after their summer IPPE and were included in the study. The average age of the students was $23.8(\mathrm{SD}=4.1)$ years. Of those, $57.8 \%$ were female and the majority
(81.9\%) were white. Average cumulative pharmacy school grade point average (GPA) for these students was 3.1 $(\mathrm{SD}=0.6)$ out of 4.0. A total of 951 codes (including codes and sub-codes) were applied to the 116 reflections. For ease of presentation, these codes were organized into six overarching themes. Below we describe each overarching theme in detail and provide the number of coded segments falling under each theme. Examples of excerpts from student reflections are reported in Appendix 1.

The first overarching theme relates to an instance when a student makes an acknowledgement of MI skills and/or components. Student reflections often mentioned specific motivational interviewing skills used during their IPPE. Ninety-five (81.9\%) students mentioned using motivational interviewing skills and/or components. Of these students, many mentioned reflecting the core concern of their patients $(69 \%)$, providing information to patients in a patient-centered manner $(22 \%)$, and exploring their patients' understanding (16\%). Many students stated that they believed reflecting their patients' core concerns could help them in their one-on-one experiences in the community. They mentioned the value in asking permission before providing information and then providing that information in a patient-centered manner. Additionally, many students stated that they more fully appreciated the usefulness of these skills when faced with difficult patients. Students mentioned learning that reflecting a

Table 1. Definitions of the Levels of Reflection Student Pharmacists Used During Patient-Centered Communication in an Introductory Pharmacy Practice Experience

Level 4: Behavior Change Level (Intention to Change Behavior)

Evidence of a change in perspective over a fundamental belief of the understanding of a key concept or $(\mathrm{N}=116), \%$ phenomenon. Evidence of change in behavior.

Level 3: Personal Insight/Experience Level

Theory is applied to practical situations. Situations encountered in practice were considered and successfully discussed in relationship to what has been taught. There were personal insights that went beyond book theory.

Level 2: Understanding Level (hypothetical/theoretical concepts)

Evidence of understanding of a concept or topic. Material is confined to theory. Reliance upon what was in the textbook or the lecture notes. Theory is not related to personal experiences, real-life application or practical situations.

Level 1: Non-reflection Level (cites definition of patient centered communication)

The essay shows no evidence of the participant attempting to reach an understanding of the concept or theory which underpins the topic. Material has been placed into an essay without the participant thinking seriously about it, trying to interpret the material, or forming a view. Largely reproduction, with or without adaptation of the work of others.

Level 0: Absent Level (no mention of patient centered communication)

Participant did not mention topic area in essay. 


\section{American Journal of Pharmaceutical Education 2019; 83 (8) Article 7244.}

patient's core concern in real life situations can really make a difference.

The second overarching theme was students' reflection on their philosophy of patient-centeredness. This theme referred to activities that were mentioned by the student that were considered patient-centered. Overall, the patient-centeredness theme included key concepts such as individualization and personalization to provide care to patients, recognition of patient autonomy, collaboration with the patient to solve the problem, and building rapport. Content from this theme was coded 104 times in 54 (46.6\%) student reflections. Student reflections mentioned specific instances when they felt they were particularly patient-centered or recognized that patient-centered care led to more productive interactions. Many of the students also mentioned that they believed this patient-centered way of thinking would benefit them in the future when they were talking to patients, and that they could see how talking to patients like this would be really helpful when they were practicing pharmacists.

The third overarching theme encompassed students' encounters with barriers to using PCC or motivational interviewing. This theme was coded when students expressed barriers related to using PCC or motivational interviewing in their interactions. Commonly reported barriers included lacking proficiency with motivational interviewing skills, not having the opportunity to talk with patients during their IPPE, and generally having a difficult time getting out of a provider-centered mindset. Forty-six (39.7\%) students reflected on barriers to using PCC or motivational interviewing a total of 84 times. Students reflected on the limited time available to talk to patients at their IPPE location as many of the locations were high-volume pharmacies. Others felt they did not have enough knowledge about medications to engage in patient-centered discussions with patients. Additionally, some students felt they needed more practice with their motivational interviewing skills before they would be confident to use them with real patients and situations. For example, students felt practicing motivational interviewing during their IPPE in a real-world setting was helpful, but that they still had a lot of work to do to be proficient. Several students seemingly came to the realization that motivational interviewing simply did not come naturally to everyone, but through practice, improvements could be made.

The fourth overarching theme was related to student reported benefits from use of PCC. This theme was coded when student pharmacists reflected on the positive impact of using PCC or motivational interviewing on their own development, as well as the benefits to their patients. Most students $(82.8 \%)$ reported benefits from their experiences attempting to use PCC or motivational interviewing in a patient care environment. The most frequently mentioned benefit was that they felt they learned from the experience $(78 \%)$. Additionally, benefits to the patient were reported by some students. For example, 21 (18.1\%) students mentioned that the use of PCC and motivational interviewing helped them to discover potential safety issues, while an additional $21(18.1 \%)$ students mentioned general benefits they believed patients gained from the interactions, such as changing their behavior or lowering their medication costs.

The fifth overarching theme was related to the student's perception of patient encounters. Students often described their perceptions of the encounters they had with patients and whether they thought the encounters went well or they felt they should have done something differently. Seventy-seven (66.4\%) student reflections mentioned perceptions of their encounters with their patients 154 times. Many believed that their interactions with patients went well, and some were even surprised that the motivational interviewing skills actually worked in real life. While there were many positive perceptions of their interactions with patients, some students expressed frustrations or difficulty when talking with patients. Despite these difficulties, many students believed they would get more comfortable with motivational interviewing with time and because this was their first experience in a pharmacy, some of their frustrations were expected. The sixth and final overarching theme identified in students' reflections was their demonstration of good general communication skills. Students mentioned using lay language, observing their patients to make sure they understood the information being provided in the interaction, and using open-ended questions. This theme was coded 33 times by $21(18.1 \%)$ students. These students reflected on their use of lay language as a way to make patients comfortable and ensure understanding.

In addition to qualitative analysis and determination of themes, a level of reflection assessment was conducted for each student pharmacist reflection. A description of each reflection level and the results of the level of reflection assessment are provided in Table 1. The largest percentage of students reflected at level 3. However, 15.5\% of students did not mention PCC (level 0) and 24.1\% did not demonstrate any understanding of the concept (level 1) in their reflections.

\section{DISCUSSION}

Patient-centered communication, including motivational interviewing, has been increasingly recognized as critical to patient safety. In a 2007 report from the JCPP, effective communication with patients is referred to as the 


\section{American Journal of Pharmaceutical Education 2019; 83 (8) Article 7244.}

"cornerstone of patient safety" and it encourages institutions to make effective communication a priority. ${ }^{12}$ Overall, most students perceived that they were able to successfully use PCC during their community pharmacy IPPE. Most of the reflections acknowledged specific motivational interviewing skills and components of PCC. As students were instructed to start implementing PCC and motivational interviewing in practice by simply reflecting the core concern, not surprisingly, the majority acknowledged this particular skill. Fewer acknowledged skills such as providing information in a patient-centered manner, individualization, exploring, and expressing empathy. However, many reflected on general communication skills that are vital for effective communication with patients. Additionally, when motivational interviewing is taught to health care professionals in a formal setting, students are instructed to start implementing the skills gradually ${ }^{13}$ and therefore, these reflections are evidence of appropriate, gradual implementation.

As a result of education and training in the Introduction to Patient Centered Communication course, nearly half of the student pharmacists reflected on a newly gained personal philosophy of patient-centeredness. Even students who reported struggling with various aspects of the activity or those that found practicing motivational interviewing in a real-world setting to be difficult still seemed to have an overall sense of the importance of PCC. Because patient-centered care and effective patient communication with methods such as motivational interviewing and empathy are emphasized in Standards 2 and 3 of the Accreditation Council for Pharmacy Education's 2016 Standards $^{4}$ and the American Association of Colleges of Pharmacy's Center for the Advancement of Pharmacy Education (CAPE) Educational Outcomes from $2013,{ }^{5}$ this general philosophy of patient-centeredness among students is an important finding. Additional focus on empathy in communication with patients should be explored in the future.

The barriers and challenges identified by students will help to tailor the classroom experience andpractical guidance offered to them. Despite best intentions, some students reported barriers to practicing motivational interviewing during the IPPE that included lack of time, lack of appropriate drug knowledge, slipping into a provider-centered communication style, and being slow to remember to use the MI skills. Most of the barriers reported are related to lack of experience, which highlights the need to incorporate opportunities for continued skill development in motivational interviewing and feedback throughout the curriculum. However, reporting lack of time and drug knowledge as barriers may be a simple misunderstanding of the goal of communication with patients in a patient-centered way. In other words, a student does not necessarily need to have drug knowledge to be able to listen to a patient and reflect their core concern. Nevertheless, a lack of drug knowledge may have made interacting with patients more intimidating for some. Emphasis on the very basic drug knowledge and little amount of time required to interact in a patient-centered way will hopefully reduce these barriers.

A review of reflection content is a useful tool to better understand how student pharmacists are using new material in practice. Practicing reflection has been shown to enhance critical thinking and problem-solving skills and is an essential step in students' ability to effectively apply the concepts taught in the classroom. ${ }^{14}$ As less than half of the essays included true reflection, students may need better guidance on how to reflect and compose a written reflection. This could be a result of lack of specificity in expectations for reflective content or the students' inexperience with introspection. ${ }^{15}$ Another possibility is that the reason students did not produce high-quality reflections was that we had not trained them to do so. Training in reflection has not been widely explored but has been shown to improve reflective ability. ${ }^{16}$ Implementing reflection training in the curriculum may be needed to ensure students are benefiting from these activities. Additionally, opportunities for reflection may need to be incorporated earlier in the curriculum to expose students to written reflections and set expectations for reflection-based assignments.

In addition to those mentioned throughout the discussion, the qualitative nature of this study had limitations. The reflections provided were from one cohort of student pharmacists from one college of pharmacy who completed their first year in 2015 . Therefore, the generalizability of our findings to other students is unknown. Additionally, students completed their IPPE at different community pharmacies, and the practice site to which a student was assigned may have influenced their ability to practice their motivational interviewing skills. This may have positively or negatively biased their overall experience with PCC. Future research should explore the barriers student pharmacists experience when attempting to use their PCC and motivational interviewing skills.

\section{CONCLUSION}

First-year PharmD students perceived that they successfully used PCC and motivational interviewing skills during their community pharmacy IPPE after completing their first didactic year and the Introduction to Patient Centered Communication course. Other pharmacy schools may benefit from a similar introductory-level PCC course, but activities that reiterate these skills and provide additional opportunities to practice PCC are necessary throughout the curriculum to ensure students are prepared to 


\section{American Journal of Pharmaceutical Education 2019; 83 (8) Article 7244.}

effectively communicate with patients. Barriers and challenges identified will help tailor the classroom experience and practical guidance offered to students. Prompted reflection encourages practical application and reinforcement of communication skill development. Reflection opportunities should be included early and often throughout the curriculum to ensure students understand expectations for reflection-based assignments. Additional opportunities to utilize PCC and motivational interviewing skills should be integrated throughout the curriculum to ensure future pharmacists are prepared to effectively communicate with their patients.

\section{REFERENCES}

1. Institute of Medicine Committee on Quality of Health Care in A. Crossing the quality chasm: a new health system for the 21 st century. Washington, DC: National Academies P;2001.

2. Stewart M, Brown JB, Donner A, et al. The impact of patientcentered care on outcomes. The Journal of Family Practice.

2000;49(9):796-804.

3. Haskard Zolnierek KB, DiMatteo MR. Physician communication and patient adherence to treatment: a meta-analysis. Medical Care. 2009;47(8):826-834.

4. Accreditation Council for Pharmacy Education. Accreditation standards and key elements for the professional program in pharmacy leading to the Doctor of Pharmacy degree. 2016; https://www.acpeaccredit.org/pdf/Standards2016FINAL.pdf. Accessed June 21, 2018. 5. Medina MS, Plaza CM, Stowe CD, et al. Center for the Advancement of Pharmacy Education 2013 Educational Outcomes. Am J Pharm Educ. 2013;77(8):Article 162.
6. Abughosh S, Wang X, Serna O, et al. A motivational interviewing intervention by pharmacy Students to improve medication adherence. J Manag Care Spec Pharm. 2017;23(5):549-560.

7. O'Brien CE, Stafford R, Franks AM. Establishment of a patientcentered communication course to address curricular gaps. Curr Pharm Teach Learn. 2018;10(7):933-939. doi: 10.1016/ j.cpt1.2018.04.008.

8. MAXQDA Plus 12 [computer program]. Berlin, Germany: VERBI Software; 2017.

9. Hsieh HF, Shannon SE. Three approaches to qualitative content analysis. Qual Health Res. 2005;15(9):1277-1288.

10. Castleberry AN, Payakachat N, Ashby S, et al. Qualitative analysis of written reflections during a teaching certificate program. Am J Pharm Educ. 2016;80(1):Article 10.

11. Kember D, Mckay J, Sinclair K, Wong FKY. A four-category scheme for coding and assessing the level of reflection in written work. Assess Eval High Edu. 2008;33(4):369-379.

12. The Joint Commission. "What did the doctor say?:" Improving Health Literacy to Protect Patient Safety. Oakbrook Terrace, IL. 2007.

13. Berger BA, Villaume WA. Motivational Interviewing for Health Care Professionals: A Sensible Approach. Washington, DC:

American Pharmacists Association; 2013.

14. McKauge L, Stupans I, Owen SM, Ryan G, Woulfe J. Building critical reflection skills for lifelong learning in the emergent landscape of a national registration and accreditation scheme. $J$ Pharm Pract. 2011;24(2):235-240.

15. Koole S, Dornan T, Aper L, et al. Factors confounding the assessment of reflection: a critical review. BMC Med Educ. 2011;11. 16. Aronson L, Niehaus B, Hill-Sakurai L, Lai C, O'Sullivan PS. A comparison of two methods of teaching reflective ability in year 3 medical students. Med Educ. 2012;46(8):807-814. 


\section{American Journal of Pharmaceutical Education 2019; 83 (8) Article 7244.}

Appendix 1. Excerpts From Student Pharmacists' Reflections That Demonstrate Overarching Themes in Patient-centered Communication

\section{Acknowledgement Acknowledgement of MI Skills and/or Components}

"I learned that patients aren't always going to come right out and ask about their core concern, and I should be looking into what they are asking to understand what it is that they are really wanting to know."

"I asked if I could give her some examples of how she could remember to take her medication, she agreed, and we continued to discuss what would be the best options for her to not forget her medication."

"This wasn't a hard situation to figure out what the problem was, but once I restated the problem to the patient, they instantly felt that I understood them and had a sense of relief."

"I learned that reflecting the core concern in real life situations makes a difference."

Philosophy of Patient-Centeredness

"I realize that some patients may be embarrassed to admit why they do not want to adhere [to their medications] or work with us, and that I should be as genuine, understanding, and kind as I can be."

"Every patient and situation is different, so the most important thing that can be done during a conversation is listen to the patient and personalize the conversation to fit their style and need the best."

"What I learned is that all patients are different and must be treated with the utmost respect and you can get to the bottom of the problem and find a solution using patient centered communication."

\section{Barriers to Using PCC or MI}

"It would be nearly impossible for [PHARMACIST] to engage in patient-centered communication and still have time to do the rest of their job."

"Some medications I was unfamiliar with so I was unable to say much about them, however I hope to learn more about them throughout my pharmacy career."

“. . I have a long way to go but I feel like practicing this skill during my rotation and at work has made me better but I still have a long way to go!"

\section{Benefits from Use of PCC}

"I learned that if you treat [patients] with respect and not act like a know-it-all, they are apt to respond in a polite manner."

"I learned to always keep in mind that patients may be insecure about filling medications with sensitive indications and that reflecting their concern will go a long way to reassure them."

"Instead of just telling her "bye" after she said "never mind, I don't want it anymore," we discussed different options so that she can get the medication that she needs and at a price much more reasonable for her."

"I asked some more information and we realized that the problem was that the medication was right but the strength wasn't."

"The [conversation] went well in that the patient decided on their own that the medication could be worth trying again after I explained that it can take longer than a week to produce the desired effects."

Perceptions of Patient Encounters

"The patient was surprised and appreciative after I reflected their concerns back to them... reflecting the core concern is actually useful in the real world!"

"The whole encounter went well because the woman was very receptive after we discussed the different options of how she could remember to take her medication."

"The [patient] looked at me [after reflecting their concern] like I had an arm growing out of my head. It was awkward. At that point I wanted to hide behind the pharmacist."

"Some patients just weren't very open and it was hard to communicate and help them."

"I got a little frustrated that the patient did not understand my explanation right away..."

\section{Demonstration of Good General Communication Skills}

"My patient taught me the importance of simplifying your language and using analogies so that they have a better understanding of their condition and how their prescription can fix the problem."

“. . the best way to approach the conversations were to gently start the conversation and talk slowly and controlled, rather than just spit information out at once." 